\title{
Interval observer for a class of uncertain nonlinear singular systems
}

\author{
G. Zheng ${ }^{\mathrm{a}, \mathrm{b}, \mathrm{c}}$, D. Efimov ${ }^{\mathrm{b}, \mathrm{c}, \mathrm{d}}$, F. J. Bejarano ${ }^{\mathrm{e}}$, W. Perruquetti ${ }^{\mathrm{b}, \mathrm{c}}$, H. Wang ${ }^{\mathrm{a}, 1}$ \\ ${ }^{a}$ School of Automation. Nanjing University of Science and Technology, 210094 Nanjing, China \\ ${ }^{b}$ Non-A, INRIA Lille-Nord Europe, 40 Avenue Halley, 59650 Villeneuve d'Ascq, France \\ ${ }^{c}$ CRIStAL, CNRS UMR 9189, Ecole Centrale de Lille, BP 48, 59651 Villeneuve d'Ascq, France. \\ ${ }^{d}$ Department of Control Systems and Informatics, National Research University, 49 Kronverkskiy av., 197101 Saint Petersburg, Russia \\ e SEPI, ESIME Ticomán, IPN, Av. San José Ticomán 600, C.P. 07340, Mexico City, Mexico.
}

\begin{abstract}
This paper investigates the problem of observer design for a general class of linear singular time-delay systems, in which the time delays are involved in the state, the output and the known input (if there exists). The involvement of the delay could be multiple which however is rarely studied in the literature. Sufficient conditions are proposed which guarantees the existence of a Luenberger-like observer for the general system.
\end{abstract}

Keywords: Delayed singular systems, commensurate delays, observer.

\section{Introduction}

Singular system approach (known as well descriptor system/algebraic-differential system) was introduced to model a large class of systems in many different domains, such as physical, biological, and economic ones, for which the standard representation sometimes cannot be applied Campbell (1982, 1980). The structure of this type of systems contains both the dynamic equations and the algebraic ones, and due to this characteristic, many well-defined concepts dealing with the observability problem for regular (non-singular) systems have to be reconsidered. In Yip and Sincovec (1981), the authors have studied the solvability, controllability and observability concepts for singular systems with regular matrix pencil. The algebraic duality between controllability and observability for singular systems with regular matrix pencil is proven by using the Schwartz distribution framework in Cobb (1984). In Hou and Muller (1999a), the concept of causal observability was proposed for singular systems. The strong observability and strong detectability of a general class of singular linear systems with unknown inputs are recently tackled in Bejarano et al. (2011, 2013) by converting the singular system into a regular one with unknown inputs and algebraic constraints. The observability problem for nonlinear singular system has been treated in Bejarano et al. (2012, 2015).

Concerning the observer design, a Luenberger-like observer has been proposed in Paraskevopoulos and Koumboulis (1992) for linear singular systems. Darouach and Boutayeb (1995)

\footnotetext{
This paper was supported in part by Ministry of Higher Education and Research Nord-Pas de Calais Regional Council, by FEDER through the Contrat de Projets Etat Region (CPER), by ARCIR Project ESTIREZ Nord-Pas de Calais Regional Council, by Project GEC-Beihang 2015, Laboratoire international associé, by Chinses NSF(61304077), and by International Science \& Technology Cooperation Program of China 2015, 2016.

${ }^{1}$ Corresponding author: hp.wang@njust.edu.cn.
}

gave necessary and sufficient condition for the existence of a reduced order observer for the linear singular systems with known inputs, and the result was extended to treat the linear singular systems with unknown inputs in Darouach et al. (1996). In Hou and Muller (1999b), a generalized observer was studied by involving the derivative of input and output. For linear singular systems with unknown inputs, a proportional-integral observer was proposed in Koenig and Mammar (2002), and its extension by involving multiple integrations to design an unknown input observer was studied in Koenig (2005). For the nonlinear singular system, Kaprielian and Turi (1992) studied an observer for a class of nonlinear singular systems in which the system was linearized around the equilibrium point. The same technique was used in Boutayeb and Darouach (1995) to study a reduced order observer for a class of nonlinear singular systems. Other techniques, such as LMI (Lu and Ho (2006); Darouach and BoutatBaddas (2008b)) and convex optimization Koenig (2006), are proposed as well to design an observer for nonlinear singular systems with known (or unknown) inputs. And recently, the technique of regularization by applying the geometrical differential method to the nonlinear singular systems was introduced in Boutat et al. (2012).

Most of the cited references are for the asymptotic estimation of the state for singular systems without uncertainties. The observer design becomes complicated when considering the systems with uncertain terms in the state and in the measurement. In this situation, the exact estimation may be not possible, and one solution is to provide the upper and lower bound estimation of the admissible values for the state by applying the theory of set-membership or interval estimation Gouzé et al. (2000); Mazenc and Bernard (2010); Raïssi et al. (2012); Efimov et al. (2012).

Concerning the interval observer design for singular system, the only work existing in the literature is Efimov et al. 
(2014), where the problem of interval observer design is addressed for a class of linear singular systems with delays. In this work, the studied singular system was decoupled into two parts $x=\left(x_{1}, x_{2}\right)^{T}$ : the dynamical one and the algebraic one. The authors then proposed an interval observer by restrictively assuming that $x_{2}$ can be fully described as the function of $x_{1}$. Thus the algebraic equation can be removed and the studied singular system is equivalent to a regular one. Without imposing this restrictive condition, this paper proposes a method to design an interval observer for a class of nonlinear singular systems with uncertainties. By imposing the observability rank condition for the linear part and assuming some boundedness properties of the studied system, this paper shows that an interval observer for this class of uncertain nonlinear singular systems always exists, which provides the upper and the lower estimations of the system's state.

In this paper, the following notation is used. $\mathbb{R}$ denotes the set of real numbers and $\mathbb{R}_{+}=\{x \in \mathbb{R}: x \geq 0\}$. $a \mathscr{R} b$ represents the element-wise relation $\mathscr{R}$ ( $a$ and $b$ are vectors or matrices): for example $a<b$ (vectors) means $\forall i: a_{i}<b_{i}$. For a matrix $\mathscr{A} \in \mathbb{R}^{m \times n}$, define $\mathscr{A}^{+}=\max \{0, \mathscr{A}\}^{2}$ and $\mathscr{A}^{-}=\mathscr{A}^{+}-\mathscr{A}$. For a vector $x \in \mathbb{R}^{n}$, define $x^{+}=\max \{0, x\}$ and $x^{-}=x^{+}-x$. For a matrix (function) $\mathscr{A}$ the symbol $\mathscr{A}_{i}$ denotes its $i^{\text {th }}$ column, for a vector (function) $b$ the symbol $b_{i}$ denotes its corresponding element. A matrix $\mathscr{A} \in \mathbb{R}^{n \times n}$ is called Metzler if all its elements outside the main diagonal are nonnegative.

\section{Problem statement and backgrounds}

\subsection{Problem statement}

Consider the following uncertain nonlinear singular system:

$$
\Sigma_{\xi}:\left\{\begin{array}{l}
\bar{E} \dot{\xi}=\bar{A} \xi+\bar{f}(\xi, u)+v(t) \\
y=\bar{C} \xi+w(t)
\end{array}\right.
$$

where $\xi \in \mathbb{R}^{n}$ is the state whose initial value belongs to a compact set $I_{0}\left(\xi\left(t_{0}\right)\right)=\left[\xi\left(t_{0}\right), \bar{\xi}\left(t_{0}\right)\right] ; y \in \mathbb{R}^{p}$ and $u \in \mathbb{R}^{m}$ are respectively the output and the input. $\bar{E} \in \mathbb{R}^{n \times n}, \bar{A} \in \mathbb{R}^{n \times n}, \bar{C} \in \mathbb{R}^{p \times n}$, and the vector field $\bar{f}$ represents the nonlinear term with the appropriate dimension. $w(t)$ and $v(t)$ are the disturbance in the output and in the model, respectively.

When the matrix $\bar{E}$ is nonsingular, then (1) can be written as:

$$
\left\{\begin{array}{l}
\dot{\xi}=\bar{E}^{-1} \bar{A} \xi+\bar{E}^{-1} \bar{f}(\xi, u)+\bar{E}^{-1} v(t) \\
y=\bar{C} \xi+w(t)
\end{array}\right.
$$

which becomes a classical regular system. We can cite lots of existing methods to design an observer for the system without the uncertainties, if it is observable. For the case with uncertainties in the state and in the output, an interval observer was proposed in Raïssi et al. (2012) for the observable system (2) to give the upper and lower estimation of the real state with the only requirement of the uncertainty boundedness. This method

\footnotetext{
${ }^{2}$ It means each element of $\mathscr{A}^{+}$is the maximum value between 0 and the maximum value of $\mathscr{A}$ at the same position.
}

is extended in Zheng et al. $(2013,2016)$ to treat the case even if the system is not observable. When the matrix $\bar{E}$ is singular, (1) represents a large class of nonlinear singular systems with uncertainties in the state and in the output, covering those studied in Koenig (2006) and Darouach and Boutat-Baddas (2008a). This paper is devoted to designing an interval observer for this larger class of uncertain nonlinear singular systems. Before this, let us firstly recall the basic background on comparison systems which will be used for interval observer design.

\subsection{Background on comparison systems}

When dealing with a qualitative property involving solutions of a complex system, it is sometimes of interest to obtain a simpler system whose solutions overvalue the solutions of the initial system in some sense. For ODE, the contributions of Müller (1926); Kamke (1932); Wazewski (1950) are probably the most important in this field: they give necessary and sufficient hypotheses ensuring that the solution of $\dot{x}=f(t, x)$, with initial state $x_{0}$ at time $t_{0}$ and function $f$ satisfying the inequality $f(t, x) \leq g(t, x)$ is overvalued by the solution of the so-called "comparison system" $\dot{z}=g(t, z)$, with initial state $z_{0} \geq x_{0}$ at time $t_{0}$, or, in other words, conditions on function $g$ that ensure $x(t) \leq z(t)$ for $t \geq t_{0}$. These results were extended to many different classes of dynamical systems Bitsoris (1978); Dambrine et al. (1995); Grujic̀ et al. (1987); Perruquetti et al. (1995b,a); Perruquetti and Richard (1996); Borne et al. (2003). From these results one can deduce the following proposition:

Proposition 1. Smith (1995) Assume that A is a Metzler matrix, $b(t) \in \mathbb{R}_{+}^{n}, \forall t \geq t_{0}$, where $t_{0}$ represents the initial time such that the system:

$$
\frac{d x(t)}{d t}=A x+b(t)
$$

possesses, for every $x\left(t_{0}\right) \in \mathbb{R}_{+}^{n}$, a unique solution $x(t)$ for all $t \geq t_{0}$. Then, for any $x\left(t_{0}\right) \in \mathbb{R}_{+}^{n}$, the inequality $x(t) \geq 0$ holds for every $t \geq t_{0}$.

In other words, under conditions of Proposition $1, \mathbb{R}_{+}^{n}$ is positively invariant w.r.t. (3). Therefore, there are two important issues when designing an interval observer for the uncertain nonlinear singular system (1): find the Metzler matrix and bound the positive term, which will be discussed in the following sections.

\section{Assumptions and Preliminary results}

Concerning the observability for singular systems, there exist several different definitions in the literature, including observability, R-observability and Impulse-observability Yip and Sincovec (1981); Dai (1989). Generally speaking, they characterize the state reconstruction ability from different aspects: R-observability defines the ability to estimate the reachable set of the studied system; Impulse-observability corresponds to the ability to estimate the impulse term of the studied system and the observability covers both mentioned abilities to estimate all states of the studied system. 
In this paper, for the uncertain nonlinear singular system $\Sigma_{\xi}$, we are interested in the interval estimation of the (nonimpulsive) trajectory of the state $\xi(t)$ with the known information of $y(t)$ and $u(t)$ for $t>0$. Therefore, this paper considers the following definition of observability adopted from Definition 1 in Bejarano et al. (2013).

Definition 1. System $\Sigma_{\xi}$ is observable if $y\left(\xi_{1}, t\right)=y\left(\xi_{2}, t\right)$ for all $\xi_{1}, \xi_{2} \in \mathbb{R}^{n}$ and $t>0$ implies $\xi_{1}\left(0^{+}\right)=\xi_{2}\left(0^{+}\right)$.

In what follows, we make the following assumption for the original system $\Sigma_{\xi}$.

Assumption 1. For the triple $(\bar{E}, \bar{A}, \bar{C})$ of system $\Sigma_{\xi}$ defined in (1), it is assumed that the following rank conditions:

$$
\operatorname{rank}\left[\begin{array}{c}
\bar{E} \\
\bar{C}
\end{array}\right]=n
$$

and

$$
\operatorname{rank}\left[\begin{array}{c}
s \bar{E}-\bar{A} \\
\bar{C}
\end{array}\right]=n, \forall s \in \mathbb{C}
$$

are satisfied.

Remark 1. It is worthy noting that system $\Sigma_{\xi}$ is not assumed to have a regular pencil Kaczorek (2007), i.e. there may exist some $s \in \mathbb{C}$ such that $\operatorname{det}(s \bar{E}-\bar{A})=0$, which implies that $\xi\left(\xi_{0}, t\right)$ of $\Sigma_{\xi}$ might have more than one solution. This will not cause any problem for the observability and observer study if each pair of $(y(t), u(t))$ for all $t>0$ corresponds only to one trajectory of $\xi\left(\xi_{0}, t\right)$ (see the example given in Bejarano et al. (2013)). Moreover, the rank conditions (4) and (5) are the necessary and sufficient conditions for the observability Dai (1989) of the following classical linear singular system:

$$
\left\{\begin{array}{l}
\bar{E} \dot{\xi}=\bar{A} \xi \\
y=\bar{C} \xi
\end{array}\right.
$$

Therefore, this paper assumes as well that these two rank conditions are satisfied for the studied uncertain nonlinear singular system (1).

It is obvious that, even if the rank conditions (4) and (5) are assumed to be satisfied, the studied uncertain nonlinear singular system $\Sigma_{\xi}$ might not be observable, due to the nonlinear term $\bar{f}(\xi, u)$ and the uncertainties $(v, w)$. And this motivates the study of the interval estimation treated in this paper. Without loss of generalities, for the singular matrix $\bar{E} \in \mathbb{R}^{n \times n}$, it is assumed that $\operatorname{rank} \bar{E}=q<n$. Due to the rank condition (4), there exists a non-singular matrix $P=\left[\begin{array}{ll}P_{1} & P_{2} \\ P_{3} & P_{4}\end{array}\right] \in \mathbb{R}^{(p+n) \times(p+n)}$ such that $P\left[\begin{array}{c}\bar{E} \\ \bar{C}\end{array}\right]=\left[\begin{array}{c}I_{n} \\ 0\end{array}\right]$, which is equivalent to:

$$
\left\{\begin{array}{l}
P_{1} \bar{E}+P_{2} \bar{C}=I_{n} \\
P_{3} \bar{E}+P_{4} \bar{C}=0
\end{array}\right.
$$

with $P_{1} \in \mathbb{R}^{n \times n}, P_{2} \in \mathbb{R}^{n \times p}, P_{3} \in \mathbb{R}^{p \times n}$ and $P_{4} \in \mathbb{R}^{p \times p}$. Based on the above result, we have the following lemma.
Lemma 1. Suppose Assumption 1 is satisfied for the triple $(\bar{E}, \bar{A}, \bar{C})$ of $\Sigma_{\xi}$. Then there always exist matrices $K \in \mathbb{R}^{n \times p}$, $L \in \mathbb{R}^{n \times p}$ and an invertible matrix $Q \in \mathbb{R}^{n \times n}$ such that the following matrix:

$$
R=Q N Q^{-1}
$$

is Hurwitz and Metzler, where

$$
N=\left(P_{1}+K P_{3}\right) \bar{A}+L \bar{C},
$$

with $P_{1}$ and $P_{3}$ being defined in (6).

Proof. As we have explained that, if (4) is satisfied, then there exists an invertible matrix $P=\left[\begin{array}{ll}P_{1} & P_{2} \\ P_{3} & P_{4}\end{array}\right]$ such that equation (6) is satisfied. Hence, for any $s \in \mathbb{C}$, we have

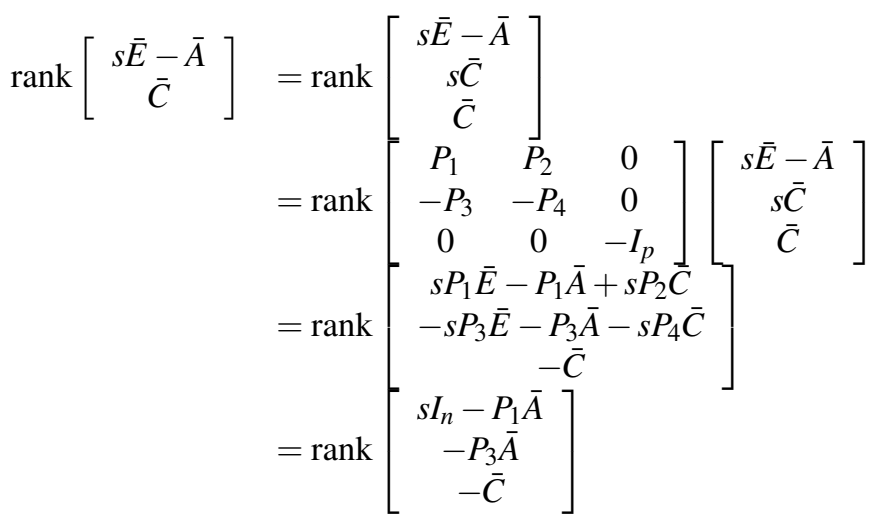

Therefore, in view of (5), we have rank $\left[\begin{array}{c}s I_{n}-P_{1} \bar{A} \\ -P_{3} \bar{A} \\ -\bar{C}\end{array}\right]=n$, for any $s \in \mathbb{C}$, which implies that the pair $\left(P_{1} \bar{A},\left[\begin{array}{c}P_{3} \bar{A} \\ \bar{C}\end{array}\right]\right)$ is observable. Thus one can find the matrices $K$ and $L$ with appropriate dimension such that

$$
N=P_{1} \bar{A}+[K, L]\left[\begin{array}{c}
P_{3} \bar{A} \\
\bar{C}
\end{array}\right]=\left(P_{1}+K P_{3}\right) \bar{A}+L \bar{C}
$$

is Hurwitz. Following Lemma 1 in Raïssi et al. (2012), we can choose a Metzler matrix $R$ with the same eigenvalues as $N$, then there always exists an invertible matrix $Q$ such that the matrix $R=Q N Q^{-1}$ is Hurwitz and Metzler.

The above matrix $Q$ was introduced to transform the Hurwitz matrix $N$ to the Hurwitz and Metzler matrix $R$. Inserting (8) into (7), we have

$$
R=Q\left(P_{1}+K P_{3}\right) \bar{A} Q^{-1}+Q L \bar{C} Q^{-1}
$$

which contains $\bar{A} Q^{-1}$ and $\bar{C} Q^{-1}$. For the sake of simplification, let us introduce a linear transformation $x=Q \xi$, with which system $\Sigma_{\xi}$ in (1) can be rewritten as follows:

$$
\Sigma_{x}:\left\{\begin{array}{l}
E \dot{x}=A x+f(x, u)+v(t) \\
y=C x+w(t)
\end{array}\right.
$$

where $E=\bar{E} Q^{-1}, A=\bar{A} Q^{-1}, C=\bar{C} Q^{-1}$ and $f(x, u)=$ $\bar{f}\left(Q^{-1} x, u\right)$. As we can see that, with such a transformation, the 
expression of $R$ can be simplified as $R=Q\left(P_{1}+K P_{3}\right) A+Q L C$, and this will simplify the observer design procedure. For the transformed system (9), we can state the following lemma.

Lemma 2. Suppose Assumption 1 is satisfied for the triple $(\bar{E}, \bar{A}, \bar{C})$ of $\Sigma_{\xi}$. Then for the transformed system $\Sigma_{x}(E, A, C)$ defined in (9), there always exist a matrix $K \in \mathbb{R}^{n \times p}$, two invertible matrices $P=\left[\begin{array}{ll}P_{1} & P_{2} \\ P_{3} & P_{4}\end{array}\right] \in \mathbb{R}^{(p+n \times(p+n)}$ and $Q \in \mathbb{R}^{n \times n}$, such that the following equality is satisfied:

$$
Q P_{24} C+Q P_{13} E=I_{n}
$$

where the matrices $P_{13}$ and $P_{24}$ are defined as follows:

$$
\begin{aligned}
& P_{13}=P_{1}+K P_{3} \\
& P_{24}=P_{2}+K P_{4}
\end{aligned}
$$

\section{Proof.}

As we have proved in Lemma 1 that if Assumption 1 is satisfied for the triple $(\bar{E}, \bar{A}, \bar{C})$ of $\Sigma_{\xi}$, then one can always find matrices $K \in \mathbb{R}^{n \times p}, L \in \mathbb{R}^{n \times p}$, and two non-singular matrices $P=\left[\begin{array}{ll}P_{1} & P_{2} \\ P_{3} & P_{4}\end{array}\right] \in \mathbb{R}^{(p+n) \times(p+n)}$ and $Q \in \mathbb{R}^{n \times n}$ such that $N$ defined in (8) is Hurwitz, and $R$ defined in (7) is Hurwitz and Metzler. By keeping the same matrices $K, P$ and $Q$ as those found in Lemma 1 , since $Q$ is invertible, then (6) is equivalent to:

$$
\begin{aligned}
& Q P_{1} \bar{E} Q^{-1}+Q P_{2} \bar{C} Q^{-1}=Q P_{1} E+Q P_{2} C=I_{n} \\
& P_{3} \bar{E} Q^{-1}+P_{4} \bar{C} Q^{-1}=P_{3} E+P_{4} C=0
\end{aligned}
$$

By noting $P_{24}=P_{2}+K P_{4}$, the above equalities yield

$$
\begin{aligned}
Q P_{24} C-I_{n} & =Q P_{2} C-I_{n}+Q K P_{4} C \\
& =-Q\left[P_{1}+K P_{3}\right] E \\
& =-Q P_{13} E
\end{aligned}
$$

where $P_{13}=P_{1}+K P_{3}$, and this ends the proof.

Since the initial condition $\xi_{0}$ for (1) is supposed to be located into a certain interval $I\left(\xi_{0}\right)=\left[\xi_{0}, \bar{\xi}_{0}\right]$, then using the diffeomorphism $x=Q \xi$, the initial condition $x_{0}$ is also known within a certain interval $I\left(x_{0}\right)=\left[\underline{x}_{0}, \bar{x}_{0}\right]$. Thus the interval estimation of $\xi$ in (1) is equivalent to estimate the interval of $x$ in (9) by using the knowledge of $(u, y)$.

\section{Main results}

This section will firstly analyze the boundedness properties by making some assumptions for (1), then propose an interval observer to estimate the state.

\subsection{Interval estimation}

Prior to introduce the interval observer for (1), let us firstly make the following assumption which is necessary in the sequel.

Assumption 2. For the studied system (1), it is assumed that:
1) the state $\xi(t)$ is bounded under the bounded input $u(t) \in$ $C^{\infty}$, i.e. $\xi(t) \in \Omega \subset \mathbb{R}^{n}$ for a given $u(t) \in \mathscr{U} \subset \mathbb{R}^{m}$ for all $t \geq 0$ where $\Omega$ and $\mathscr{U}$ are two given compact sets;

2) the function $\bar{f}(\xi, u)$ for all $\xi \in \Omega$ and $u \in \mathscr{U}$ is locally Lipschtiz w.r.t $(\xi, u)$;

3) the disturbances $w(t)$ and $v(t)$ are bounded, and the derivative of $w(t)$ is bounded for all $t \geq 0$, i.e. there exist constants $\bar{v}, \underline{v}, \bar{w}, \underline{w}, \overline{w_{d}}, \underline{w_{d}}$ such that $\underline{v} \leq v(t) \leq \bar{v}$, $\underline{w} \leq w(t) \leq \bar{w}$, and $\underline{w_{d}} \leq \dot{w}(t) \leq \overline{w_{d}}$.

Remark 2. Since system (9) was transformed from the system (1) by applying the diffeomorphism $x=Q \xi: \Omega \rightarrow \mathscr{X}$, therefore if Assumption 2 is fulfilled for (1), then the state $x(t)$ of (9) is bounded as well for the bounded input $u(t)$, i.e. $x(t) \in \mathscr{X} \subset \mathbb{R}^{n}$ for all $t \geq 0$ where $\mathscr{X}$ represents the compact set defined via the diffeomorphism. Moreover, since $f(x, u)=\bar{f}\left(Q^{-1} x, u\right)$ in (9), thus it is locally Lipschtiz w.r.t $(x, u)$ for all $x \in \mathscr{X}$ and $u \in \mathscr{U}$.

With Assumption 2, let us recall the following boundedness results stated in Zheng et al. $(2013,2016)$ and Efimov et al. (2012) which will be used to design the interval observer.

Lemma 3. Zheng et al. (2016) For a locally Lipschitz continuous function $f(x, u)$ w.r.t $(x, u)$ with $x \in \mathscr{X}$ and $u \in \mathscr{U}$, there exist two functions $\bar{f}, f: \mathbb{R}^{2 n+m} \rightarrow \mathbb{R}^{n}$ such that, for $\underline{x} \leq x \leq \bar{x}$ with $x \in \mathscr{X}$, the following inequalities hold:

$$
\underline{f}(\underline{x}, \bar{x}, u) \leq f(x, u) \leq \bar{f}(\underline{x}, \bar{x}, u)
$$

and for a given submultiplicative norm $\|\cdot\|$ we have

$$
\begin{aligned}
&\|\bar{f}(\underline{x}, \bar{x}, u)-f(x, u)\| \leq \bar{l}_{\bar{f}}\|\bar{x}-x\|+\underline{l}_{\bar{f}}\|\underline{x}-x\|+l_{\bar{f}} \\
&\|\underline{f}(\underline{x}, \bar{x}, u)-f(x, u)\| \leq \bar{l}_{f}\|\bar{x}-x\|+\underline{l}_{\underline{f}}\|\underline{x}-x\|+l_{f}
\end{aligned}
$$

for some positive constants $\bar{l}_{\bar{f}}, \underline{l}_{\bar{f}}, l_{\bar{f}}, \bar{l}_{f}, \underline{l}_{f}$ and $\underline{l}_{\underline{f}}$.

Lemma 4. Efimov et al. (2012) Let $x \in \mathscr{X} \subset \mathbb{R}^{n}$ be a vector variable with $\underline{x} \leq x \leq \bar{x}$, where $\underline{x}, \bar{x} \in \mathscr{X}$ :

1) If $\mathscr{A} \in \mathbb{R}^{m \times n}$ is a constant matrix, then

$$
\mathscr{A}^{+} \underline{x}-\mathscr{A}^{-} \bar{x} \leq \mathscr{A} x \leq \mathscr{A}^{+} \bar{x}-\mathscr{A}^{-} \underline{x}
$$

2) If $\mathscr{A} \in \mathbb{R}^{m \times n}$ is a matrix variable, with $\underline{\mathscr{A}} \leq \mathscr{A} \leq \overline{\mathscr{A}}$ for some $\underline{\mathscr{A}} \in \mathbb{R}^{m \times n}, \overline{\mathscr{A}} \in \mathbb{R}^{m \times n}$, then

$$
\begin{gathered}
\underline{\mathscr{A}}^{+} \underline{x}^{+}-\overline{\mathscr{A}}^{+} \underline{x}^{-}-\underline{\mathscr{A}}^{-} \bar{x}^{+}+\overline{\mathscr{A}}^{-} \bar{x}^{-} \leq \mathscr{A} x \\
\leq \overline{\mathscr{A}}^{+} \bar{x}^{+}-\underline{\mathscr{A}}^{+} \bar{x}^{-}-\overline{\mathscr{A}}^{-} \underline{x}^{+}+\underline{\mathscr{A}}^{-} \underline{x}^{-}
\end{gathered}
$$

As we have proved in Section 3, if Assumption 1 and Assumption 2 are satisfied, then there exist the matrices $K, L$, and the non-singular matrices $P$ and $Q$ such that the matrix $N$ defined in (8) is Hurwitz, and the matrix $R$ defined in (7) is Hurwitz and Metzler. For the sake of simplicity, note

$$
Q_{P_{13}}=Q P_{13}, Q_{P_{24}}=Q P_{24}, Q_{L}=Q L
$$


where $P_{13}$ and $P_{24}$ are defined in (10). Then one can design the following two dynamics:

$$
\left\{\begin{array}{l}
\dot{\bar{z}}=R \bar{z}+\left(R Q_{P_{24}}-Q_{L}\right) y+\bar{\Delta}(\underline{z}, \bar{z}, u) \\
\bar{x}=\bar{z}+Q_{P_{24}} y
\end{array}\right.
$$

and

$$
\left\{\begin{array}{l}
\dot{\dot{z}}=R \underline{z}+\left(R Q_{P_{24}}-Q_{L}\right) y+\underline{\Delta}(\underline{z}, \bar{z}, u) \\
\underline{x}=\underline{z}+Q_{P_{24}} y
\end{array}\right.
$$

with

$$
\begin{aligned}
& \bar{\Delta}(z, \bar{z}, u)=\overline{\Delta_{f}}(\underline{z}, \bar{z}, u)+\overline{\Delta_{v}}+\overline{\Delta_{w}}+\overline{\Delta_{w_{d}}} \\
& \overline{\Delta_{f}}(\underline{z}, \bar{z}, u)=Q_{P_{13}}^{+} \bar{f}(\underline{z}, \bar{z}, u)-Q_{P_{13}} \underline{f}(\underline{z}, \bar{z}, u) \\
& \overline{\Delta_{v}}=Q_{P_{13}}^{+} \bar{v}-Q_{P_{13}}^{-} \underline{v} \\
& \overline{\Delta_{w}}=Q_{L}^{+} \bar{w}-Q_{L}^{-} \underline{w} \\
& \overline{\Delta_{w_{d}}}=Q_{P_{24}}^{-} \overline{w_{d}}-Q_{P_{24}}^{+} \underline{w_{d}}
\end{aligned}
$$

and

$$
\begin{aligned}
& \underline{\Delta}(\underline{z}, \bar{z}, u)=\Delta_{f}(\underline{z}, \bar{z}, u)+\underline{\Delta_{v}}+\underline{\Delta_{w}}+\Delta_{w_{d}} \\
& \Delta_{f}(\underline{z}, \bar{z}, u)=Q_{P_{13}}^{+} \underline{f}(\underline{z}, \bar{z}, \bar{u})-Q_{P_{13}}^{-} \bar{f} \frac{(\underline{z}, \bar{z}, u)}{\overline{\Delta_{v}}}=Q_{L}^{+} \underline{v}-Q_{L}^{-} \bar{v} \\
& \overline{\Delta_{w}}=Q_{L}^{+} \underline{w}-Q_{L}^{-} \bar{w} \\
& \underline{\Delta_{w_{d}}}=Q_{P_{24}}^{-} \underline{w_{d}}-Q_{P_{24}}^{+} \overline{w_{d}}
\end{aligned}
$$

Let us remark that the proposed observer is of a more general form, implicitly covers the conventional way by injecting the observation error signals, such as Luenberger observer. In fact, due to the singularity of the matrix $E$, the conventional observer cannot be applied directly to design observers for singular systems.

By noting

$$
\begin{aligned}
\bar{\Gamma}(\underline{x}, \bar{x}, x, u)= & \bar{\Delta}(\underline{z}, \bar{z}, u)-Q_{P_{13}} f(x, u)-Q_{P_{13}} v(t) \\
& -Q_{L} w(t)+Q_{P_{24}} \dot{w}(t)
\end{aligned}
$$

and

$$
\begin{aligned}
\Gamma(\underline{x}, \bar{x}, x, u)= & -\underline{\Delta}(\underline{z}, \bar{z}, u)+Q_{P_{13}} f(x, u)+Q_{P_{13}} v(t) \\
& +Q_{L} w(x, u)-Q_{P_{24}} \dot{w}(t)
\end{aligned}
$$

we have the following corollary.

Corollary 1. For $\bar{\Gamma}(\underline{x}, \bar{x}, x, u)$ and $\underline{\Gamma}(\underline{x}, \bar{x}, x, u)$ defined in (13) and (14) with $x \in \mathscr{X}$ and $u \in \mathscr{U}$, we have

$$
\bar{\Gamma}(\underline{x}, \bar{x}, x, u) \geq 0 \text { and } \underline{\Gamma}(\underline{x}, \bar{x}, x, u) \geq 0
$$

Moreover, there exist positive constants $\underline{\underline{l}}_{\underline{\Gamma}}, \bar{l}_{\underline{\Gamma}}, \underline{l}_{\bar{\Gamma}}, \bar{l}_{\bar{\Gamma}}$ and $l_{\bar{\Gamma}}, l_{\underline{\Gamma}}$ such that for a chosen submultiplicative norm $\|\cdot\|$ the following inequalities:

$$
\begin{aligned}
&\|\bar{\Gamma}(\underline{x}, \bar{x}, x, u)\| \leq \bar{l}_{\bar{\Gamma}}\|\bar{x}-x\|+\underline{l}_{\bar{\Gamma}}\|x-\underline{x}\|+l_{\bar{\Gamma}} \\
&\|\underline{\Gamma}(\underline{x}, \bar{x}, x, u)\| \leq \bar{l}_{\underline{\Gamma}}\|\bar{x}-x\|+\underline{l}_{\underline{\Gamma}}\|x-\underline{x}\|+l_{\underline{\Gamma}}
\end{aligned}
$$

are satisfied for all $t \geq 0$

Proof. The proof follows directly from the definition of the functions $\bar{\Gamma}(\underline{x}, \bar{x}, x, u)$ and $\underline{\Gamma}(\underline{x}, \bar{x}, x, u)$, and the results stated in Lemma 3 and 4.
Theorem 1. Suppose Assumption 1 and Assumption 2 are satisfied. Then for any initial state $x\left(t_{0}\right)$ of (9) belongs to a certain interval $I\left(x\left(t_{0}\right)\right)=\left[\underline{x}\left(t_{0}\right), \bar{x}\left(t_{0}\right)\right]$, systems (11) and (12) form an interval observer for (9) such that the following inequality:

$$
\underline{x}(t) \leq x(t) \leq \bar{x}(t),
$$

holds for all $t \geq t_{0}$. Mover, if there exist positive definite symmetric matrices $S, M$ and a positive scalar $\mu$ such that the following LMI is satisfied:

$$
\left[\begin{array}{cc}
\mathscr{R}^{T} S+S \mathscr{R}+\frac{\alpha}{\mu} I+M & S \\
S & -\frac{1}{\mu} I
\end{array}\right] \preceq 0
$$

where $\mathscr{R}=\operatorname{diag}\{R, R\}, \alpha=2 \max \left\{\underline{l}_{\Gamma}^{2}, \bar{l}_{\Gamma}^{2}, \underline{l}_{\bar{\Gamma}}^{2}, \bar{l}_{\bar{\Gamma}}^{2}, l_{\bar{\Gamma}}^{2}, l_{\Gamma}^{2}\right\}$, then the variables $\underline{x}(t)$ and $\bar{x}(t)$ are bounded for all $t \geq 0$.

Proof. Denote $\bar{\varepsilon}=\bar{x}-x$ and $\underline{\varepsilon}=x-\underline{x}$. As we have proved, if both Assumption 1 and Assumption 2 are satisfied, there exist the matrices $K, L, P$ and $Q$ such that (1) can be transformed into (9). Then the observation errors for the proposed interval observers (11) and (12) are governed by the following dynamics:

$$
\begin{aligned}
\dot{\bar{\varepsilon}}= & \dot{\bar{z}}+Q_{P_{24}}(C \dot{x}+\dot{w}(t))-\dot{x} \\
= & R \bar{x}-Q_{L}(C x+w(t))+\bar{\Delta}(z, \bar{z}, u) \\
& +Q_{P_{24}} \dot{w}(t)+\left[Q_{P_{24}} C-I_{n}\right] \dot{x}
\end{aligned}
$$

and

$$
\begin{aligned}
\dot{\dot{\varepsilon}}= & -R \underline{x}+Q_{L}(C x+w(t))-\underline{\Delta}(\underline{z}, \bar{z}, u) \\
& -Q_{P_{24}} \dot{w}(t)-\left[Q_{P_{24}} C-I_{n}\right] \dot{x}
\end{aligned}
$$

According to Lemma 2, we have

$$
\begin{aligned}
\dot{\bar{\varepsilon}}= & R \bar{x}-Q_{L}(C x+w(t))+\bar{\Delta}(\underline{z}, \bar{z}, u)+Q_{P_{24}} \dot{w}(t)-Q_{P_{13}} E \dot{x} \\
= & R \bar{x}-Q_{P_{13}}[A x+f(x, u)+v(t)]+\bar{\Delta}(\underline{z}, \bar{z}, u) \\
& -Q_{L}(C x+w(t))+Q_{P_{24}} \dot{w}(t) \\
= & R \bar{x}-\left[Q_{L} C+Q_{P_{13}} A\right] x-Q_{P_{13}} f(x, u)-Q_{P_{13}} v(t) \\
& -Q_{L} w(t)+Q_{P_{24}} \dot{w}(t)+\bar{\Delta}(\underline{z}, \bar{z}, u) \\
= & R \bar{\varepsilon}-Q_{P_{13}} f(x, u)-Q_{P_{13}} v(t)-Q_{L} w(t) \\
& +Q_{P_{24}} \dot{w}(t)+\bar{\Delta}(\underline{z}, \bar{z}, u)
\end{aligned}
$$

where $Q_{L} C+Q_{P_{13}} A=Q\left[L \bar{C}+P_{13} \bar{A}\right] Q^{-1}=R$, and

$$
\begin{aligned}
\dot{\varepsilon}= & R \underline{\varepsilon}+Q_{P_{13}} f(x, u)+Q_{P_{13}} v(t)+Q_{L} w(x, u) \\
& -Q_{P_{24}} \dot{w}(x, u)-\underline{\Delta}(\underline{z}, \bar{z}, u)
\end{aligned}
$$

According to (13) and (14), we have

$$
\begin{aligned}
& \dot{\bar{\varepsilon}}=R \bar{\varepsilon}+\bar{\Gamma}(\underline{x}, \bar{x}, x, u) \\
& \dot{\varepsilon}=R \underline{\varepsilon}+\underline{\Gamma}(\underline{x}, \bar{x}, x, u)
\end{aligned}
$$

Based on Corollary 1, i.e. $\bar{\Gamma}(\underline{x}, \bar{x}, x, u) \geq 0$ and $\underline{\Gamma}(\underline{x}, \bar{x}, x, u) \geq 0$, since the matrix $R$ is Hurwitz and Metzler, then we can conclude according to Proposition 1 that, for any initial state $x\left(t_{0}\right) \in$ $\left[x\left(t_{0}\right), \bar{x}\left(t_{0}\right)\right]$, we always have $x(t) \leq x(t) \leq \bar{x}(t)$ for all $t \geq t_{0}$.

Moreover, note $\varepsilon=\left(\bar{\varepsilon}^{T}, \varepsilon^{T}\right)^{T}$, then the observation error system (17) can be rewritten as:

$$
\dot{\varepsilon}=\mathscr{R} \varepsilon+\Gamma(\underline{x}, \bar{x}, x, u)
$$


where $\mathscr{R}=\operatorname{diag}\{R, R\}$ and

$$
\Gamma(\underline{x}, \bar{x}, x, u)=\left[\bar{\Gamma}^{T}(\underline{x}, \bar{x}, x, u), \underline{\Gamma}^{T}(\underline{x}, \bar{x}, x, u)\right]^{T}
$$

Since the matrix $R$ is Hurwitz and Metzler, so is the matrix $\mathscr{R}$. Now consider the Lyapunov function: $V(\varepsilon)=\varepsilon^{T} S \varepsilon$ where $S$ being a positive definite symmetric matrix, then we have:

$$
\begin{aligned}
\frac{d V(\varepsilon)}{d t} & =\varepsilon^{T}\left(\mathscr{R}^{T} S+S \mathscr{R}\right) \varepsilon+2 \varepsilon^{T} S \Gamma(\underline{x}, \bar{x}, x, u) \\
& \leq \varepsilon^{T}\left(\mathscr{R}^{T} S+S \mathscr{R}\right) \varepsilon+\mu \varepsilon^{T} S^{2} \varepsilon+\frac{1}{\mu}\|\Gamma(\underline{x}, \bar{x}, x, u)\|^{2}
\end{aligned}
$$

According to Corollary 1 , there exists a positive constant $\alpha$ such that

$$
\|\Gamma(\underline{x}, \bar{x}, x, u)\|^{2} \leq \alpha\left(\|\varepsilon\|^{2}+1\right)
$$

where $\alpha=2 \max \left\{\underline{l_{\underline{\Gamma}}}, \bar{l}_{\underline{\Gamma}}^{2}, \underline{l}_{\bar{\Gamma}}^{2}, \bar{l}_{\bar{\Gamma}}^{2}, l_{\bar{\Gamma}}^{2}, l_{\underline{\Gamma}}^{2}\right\}$, thus (18) can be written as:

$$
\frac{d V(\varepsilon)}{d t} \leq \varepsilon^{T}\left(\mathscr{R}^{T} S+S \mathscr{R}+\mu S^{2}+\frac{1}{\mu} \alpha I\right) \varepsilon+\alpha
$$

Therefore, if there exist positive definite symmetric matrices $S$, $M$ and a positive scalar $\mu$ such that the LMI defined in (16) is satisfied, then we obtain:

$$
\frac{d V(\varepsilon)}{d t} \leq-\varepsilon^{T} M \varepsilon+\alpha
$$

which implies that the variables $\underline{x}(t)$ and $\bar{x}(t)$ are bounded for all $t \geq 0$.

Corollary 2. For system (9) with the initial state $\xi\left(t_{0}\right) \in$ $\left[\xi\left(t_{0}\right), \bar{\xi}\left(t_{0}\right)\right]$, if Assumption 1 and Assumption 2 are satisfied, then there exists a non-singular matrix $Q$ such that the interval estimation of $\xi$ in (1) is given as follows:

$$
\left[Q^{-1}\right]^{+} \underline{x}-\left[Q^{-1}\right]^{-} \bar{x} \leq \xi \leq\left[Q^{-1}\right]^{+} \bar{x}-\left[Q^{-1}\right]^{-} \underline{x}
$$

where $\underline{x}$ and $\bar{x}$ are the states of the proposed interval observer defined in (11) and (12).

\section{Proof.}

The proof of this corollary is straightforward by applying Lemma 4 to the result stated in Theorem 1.

Let us remark that Assumption 2 imposed the state boundedness $\xi(t) \in \Omega \subset \mathbb{R}^{n}$ under a given bounded input $u(t) \in \mathscr{U} \subset$ $\mathbb{R}^{m}$ for all $t \geq 0$, and this property is sometimes difficult to be checked for general uncertain nonlinear singular systems, therefore the proposed result could not be applied. However, if the boundedness property of the state in Assumption 2 is satisfied only for $t \in[0, T]$ with $T$ being a finite time, i.e. $\xi(t) \in \Omega \subset \mathbb{R}^{n}$ under a given bounded input $u(t) \in \mathscr{U} \subset \mathbb{R}^{m}$ for all $t \in[0, T]$, then Theorem 1 is still valid during this finite time $T$. Therefore the proposed result can be relaxed by the following corollary.

Corollary 3. Suppose Assumption 1 and Assumption 2 are valid only for $t \in[0, T]$ with $T$ being a finite time. Then for any initial state $x\left(t_{0}\right)$ of (9) belongs to a certain interval
$I\left(x\left(t_{0}\right)\right)=\left[\underline{x}\left(t_{0}\right), \bar{x}\left(t_{0}\right)\right]$, systems (11) and (12) give an interval estimation of $x(t)$ of (9) for $t \in\left[t_{0}, T\right]$, i.e. $\underline{x}(t) \leq x(t) \leq \bar{x}(t)$ for all $t \in\left[t_{0}, T\right]$. And the interval estimation of $\xi(t)$ of (1) for all $t \in\left[t_{0}, T\right]$ can be still obtained by (20).

Remark 3. Although this paper considers only the nonlinear singular system with the uncertainties in the state and in the output, it is worthy noting that the proposed method can be easily extended to treat the nonlinear singular system with parameter uncertainty as well. Suppose that the unknown (may be time-varying) parameters $\theta$ belong to a compact set $\Theta \subset \mathbb{R}^{q}$, for the following nonlinear singular systems with uncertainties:

$$
\left\{\begin{array}{l}
\bar{E} \dot{\xi}=\bar{A} \xi+\bar{f}(\xi, \theta, u)+v(t) \\
y=\bar{C} \xi+w(t)
\end{array}\right.
$$

we can still use the proposed method to design the interval observer if $\bar{f}(\xi, \theta, u)$ is locally Lipschitz continuous.

\subsection{Design procedure}

For system $\Sigma_{\xi}(\bar{E}, \bar{A}, \bar{C})$ defined in (1), supposed that Assumption 1 and Assumption 2 are both satisfied for all $t \geq 0$ (or for all $t \in[0, T]$ ), then the interval estimation of the state $\xi$ for (1) can be obtained via the following procedure:

Step1: Due to the satisfaction of the rank condition (4) in Assumption 1, determine an invertible matrix $P=$ $\left[\begin{array}{ll}P_{1} & P_{2} \\ P_{3} & P_{4}\end{array}\right]$ such that $P\left[\begin{array}{c}\bar{E} \\ \bar{C}\end{array}\right]=\left[\begin{array}{c}I_{n} \\ 0\end{array}\right]$;

Step2: Due to the satisfaction of the rank condition (5) in Assumption 1 , choose the matrices $K, L$ such that $N=$ $L \bar{C}+\left(P_{1}+K P_{3}\right) \bar{A}$ is Hurwitz;

Step3: Choose an invertible matrix $Q$ such that $R=Q N Q^{-1}$ is Hurwitz and Metzler;

Step4: Transform system $\bar{\Sigma}_{\xi}(\bar{E}, \bar{A}, \bar{C})$ into $\Sigma_{x}(E, A, C)$ by applying the diffeomorphism $x=Q \xi$;

Step5: If Assumption 2 is satisfied for all $t \geq 0$ (or for all $t \in$ $[0, T])$, calculate the bound of the function $f$ in (9);

Step6: Design interval observer (11) and (12) for $\Sigma_{x}(E, A, C)$, which yields $\underline{x}(t) \leq x(t) \leq \bar{x}(t)$ for all $t \geq 0$ (or for all $t \in[0, T])$;

Step7: Finally we obtain the interval estimation of $\xi(t)$ for all $t \geq 0$ (or for all $t \in[0, T]$ ):

$$
\left[Q^{-1}\right]^{+} \underline{x}-\left[Q^{-1}\right]^{-} \bar{x} \leq \xi \leq\left[Q^{-1}\right]^{+} \bar{x}-\left[Q^{-1}\right]^{-} \underline{x} .
$$

Remark 4. It is clear that the estimation precision for the original state $\xi$ depends on the well-chosen matrix $Q$, and the good choice of $Q$ will not lead to the overestimation. This issue has been studied in Chebotarev et al. (2015) in the sense to minimize the influence of the uncertainties on the interval estimation accuracy.

\section{Examples}

\subsection{Academic example}

Let us consider the following uncertain nonlinear singular system:

$$
\begin{aligned}
& \bar{E} \dot{\xi}=\bar{A} \xi+\bar{f}(\xi, u)+v(t) \\
& y=\bar{C} \xi+w(t)
\end{aligned}
$$


with

$$
\bar{E}=\left[\begin{array}{lll}
0 & 1 & 0 \\
0 & 0 & 1 \\
0 & 0 & 0
\end{array}\right], \bar{A}=\left[\begin{array}{ccc}
20 & 0 & 0 \\
1 & 8 & 0 \\
1 & 0 & 1
\end{array}\right] \text { and } \bar{C}=\left[\begin{array}{l}
1 \\
0 \\
0
\end{array}\right]^{T}
$$

$\bar{f}(\xi, u)=\left[\begin{array}{c}\sin \left(\frac{\xi_{3}}{32}\right) \\ u \sin \left(\frac{4 \xi_{2}-\xi_{3}}{240}\right) \\ -0.75 \sin \left(\frac{4 \xi_{2} \xi_{3}-\xi_{3}^{2}}{120}\right)\end{array}\right] \quad$ and $\quad v(t)=$ $\left[\begin{array}{c}0.1 \cos 3 t \\ 0.2 \sin 4 t \\ 0.15 \sin 2 t\end{array}\right], w(t)=0.1 \sin (t)$. It is straightforward to check that the studied uncertain nonlinear singular system may be not observable with respect to the output $y$, thus an asymptotic observer could not be designed. The following applies the proposed method to design an interval observer to get the upper and the lower estimations of the state.

Step 1:

Let us firstly check the rank conditions imposed in Assumption 1. Since

$$
\operatorname{rank}\left[\begin{array}{c}
\bar{E} \\
\bar{C}
\end{array}\right]=3
$$

and

$$
\operatorname{rank}\left[\begin{array}{c}
s \bar{E}-\bar{A} \\
\bar{C}
\end{array}\right]=3, \forall s \in \mathbb{C}
$$

thus Assumption 1 is fulfilled. Moreover, the input is chosen as $u(t)=0.5 \sin (t)$ such that Assumption 2 is at least satisfied for $t \in[0, T]$ with $T$ being a finite time (see Fig. 1-3 for $T=5$ ).

Then one can find an invertible matrix $P=$ $\left[\begin{array}{llll}0 & 0 & 0 & 1 \\ 1 & 0 & 0 & 0 \\ 0 & 1 & 0 & 0 \\ 0 & 0 & 1 & 0\end{array}\right]=\left[\begin{array}{ll}P_{1} & P_{2} \\ P_{3} & P_{4}\end{array}\right]$ with $P_{1}=\left[\begin{array}{lll}0 & 0 & 0 \\ 1 & 0 & 0 \\ 0 & 1 & 0\end{array}\right]$, $P_{2}=\left[\begin{array}{l}1 \\ 0 \\ 0\end{array}\right], P_{3}=\left[\begin{array}{lll}0 & 0 & 1\end{array}\right]$ and $P_{4}=\left[\begin{array}{l}0\end{array}\right]$ such that $P\left[\begin{array}{c}\bar{E} \\ \bar{C}\end{array}\right]=\left[\begin{array}{c}I_{3 \times 3} \\ 0\end{array}\right]$.

Step 2:

Therefore, we can choose:

$$
K=[0,-1,-6]^{T}, \quad L=[-2,-19,5]^{T}
$$

such that $N=L \bar{C}+\left(P_{1}+K P_{3}\right) \bar{A}=\left[\begin{array}{ccc}-2 & 0 & 0 \\ 0 & 0 & -1 \\ 0 & 8 & -6\end{array}\right]$ with negative eigenvalues $(-2,-4,-2)$.

Step 3:

Hence, we can find the following invertible matrix:

$$
Q=\left[\begin{array}{ccc}
1 & 0 & 0 \\
0 & 1 & -0.25 \\
0 & 0 & 1
\end{array}\right]
$$

such that $R=Q N Q^{-1}=\left[\begin{array}{ccc}-2 & 0 & 0 \\ 0 & -2 & 0 \\ 0 & 8 & -4\end{array}\right]$ which is Hurwitz and Metzler.

\section{Step 4:}

So, with the transformation $x=Q \xi$, system (21) can be written as:

$$
\begin{aligned}
& E \dot{x}=A x+f(x, u)+v(t) \\
& y=C x+w(t)
\end{aligned}
$$

with $E=\bar{E} Q^{-1}=\left[\begin{array}{ccc}0 & 1 & 0.25 \\ 0 & 0 & 1 \\ 0 & 0 & 0\end{array}\right], \quad A=\bar{A} Q^{-1}=$ $\left[\begin{array}{ccc}20 & 0 & 0 \\ 1 & 8 & 2 \\ 1 & 0 & 1\end{array}\right], \quad C=\bar{C} Q^{-1}=\left[\begin{array}{lll}1 & 0 & 0\end{array}\right] \quad$ and $f(x, u)=\bar{f}\left(Q^{-1} x, u\right)=\left[\begin{array}{c}\sin \left(\frac{x_{3}}{32}\right) \\ u \sin \left(\frac{x_{2}}{60}\right) \\ -0.75 \sin \left(\frac{x_{2} x_{3}}{30}\right)\end{array}\right]$.

\section{Step 5:}

Let us now compute the bounding function for $f(x, u)$ in (22). To this end, define the following two functions:

$$
\begin{aligned}
& \operatorname{Product}(\underline{x}, \bar{x})=\left[\begin{array}{l}
\min \left\{\bar{x}_{2} \bar{x}_{3}, \underline{x}_{2} \bar{x}_{3}, \bar{x}_{2} \underline{x}_{3}, \underline{x}_{2} \underline{x}_{3}\right\} \\
\max \left\{\bar{x}_{2} \bar{x}_{3}, \underline{x}_{2} \bar{x}_{3}, \bar{x}_{2} \underline{x}_{3}, \underline{x}_{2} \underline{x}_{3}\right\}
\end{array}\right], \\
& {\left[\begin{array}{c}
\underline{\sin }(\underline{x}, \bar{x}) \\
\overline{\sin }(\underline{x}, \bar{x})
\end{array}\right]=\left[\begin{array}{l}
\sin (\underline{x}) \\
\sin (\bar{x})
\end{array}\right]}
\end{aligned}
$$

corresponding to the interval of the product $x_{2} x_{3}$ for $x=\left[x_{2} x_{3}\right]^{T}$ with $x \leq x \leq \bar{x}$ and the interval of the function $\sin (x)$ for a scalar $x$ with $\underline{x} \leq x \leq \bar{x}$ (for $|x| \leq \pi / 2$ ). Then we have the following bounding functions for $f$ :

$$
\begin{aligned}
f_{1}(\underline{x}, \bar{x}) & =\underline{\sin }(\underline{x} / 32, \bar{x} / 32) \\
\overline{\bar{f}}_{1}(\underline{x}, \bar{x}) & =\sin (\underline{x} / 32, \bar{x} / 32) \\
{\left[\underline{f}_{2}(\underline{x}, \bar{x})\right] } & =\operatorname{Product}\left([\underline{\underline{u}} \overline{\bar{u}}],\left[\frac{\overline{\sin }(\underline{x} / 60, \bar{x} / 60)}{\underline{\sin }(\underline{x} / 60, \bar{x} / 60)}\right]\right) \\
\overline{\bar{f}}_{3}(\underline{x}, \bar{x}, \bar{x}) & =-0.75 \sin (\operatorname{Product}(\underline{x} / 30, \bar{x} / 30)) \\
\bar{f}_{3}(\underline{x}, \bar{x}) & =-0.75 \underline{\sin }(\operatorname{Product}(\underline{x} / 30, \bar{x} / 30))
\end{aligned}
$$

Take $\underline{l}_{\Gamma}=\bar{l}_{\underline{\Gamma}}=\underline{l}_{\bar{\Gamma}}=\bar{l}_{\bar{\Gamma}}=l_{\bar{\Gamma}}=l_{\underline{\Gamma}}=1$, then we have $\alpha=$ 2. By solving the LMI defined in (16), we find $\mu=1, S=$ $\operatorname{diag}\left\{\mathrm{S}_{1}, \mathrm{~S}_{1}\right\}, M=\operatorname{diag}\left\{\mathrm{M}_{1}, \mathrm{M}_{1}\right\}$ with

$$
S_{1}=\left[\begin{array}{ccc}
1.6 & 0 & 0 \\
0 & 3 & 0.4 \\
0 & 0.4 & 0.5
\end{array}\right], M_{1}=\left[\begin{array}{ccc}
3.5 & 0 & 0 \\
0 & 4.3 & 0.2 \\
0 & 0.2 & 3.4
\end{array}\right] \text {, }
$$

thus all conditions of Theorem 1 have been verified.

\section{Step 6:}

Then we can design the interval observer for the transformed system (22). The results of the interval estimations of the state $x$ for this system are depicted in Fig. 1-3, from which we can see that the proposed observer can provide both the upper and the lower estimations of $x$.

Step 7:

Then, by applying

$$
\left[Q^{-1}\right]^{+} \underline{x}-\left[Q^{-1}\right]^{-} \bar{x} \leq \xi \leq\left[Q^{-1}\right]^{+} \bar{x}-\left[Q^{-1}\right]^{-} \underline{x}
$$

we obtain the upper and the lower estimation of the real state $\xi$ for the uncertain nonlinear singular systems (21) (see Fig. 4-6). 


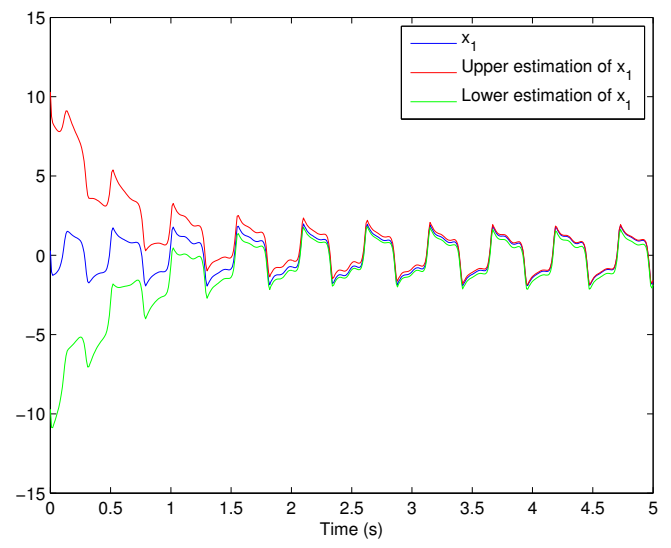

Figure 1: The interval estimation for $x_{1}$.

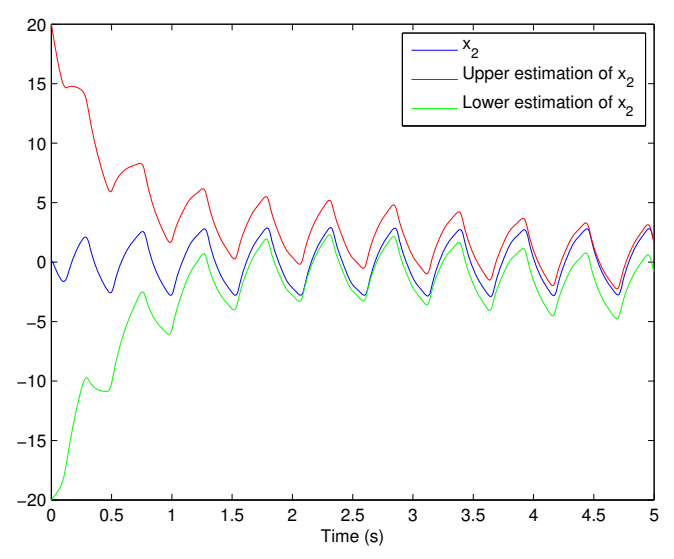

Figure 2: The interval estimation for $x_{2}$.

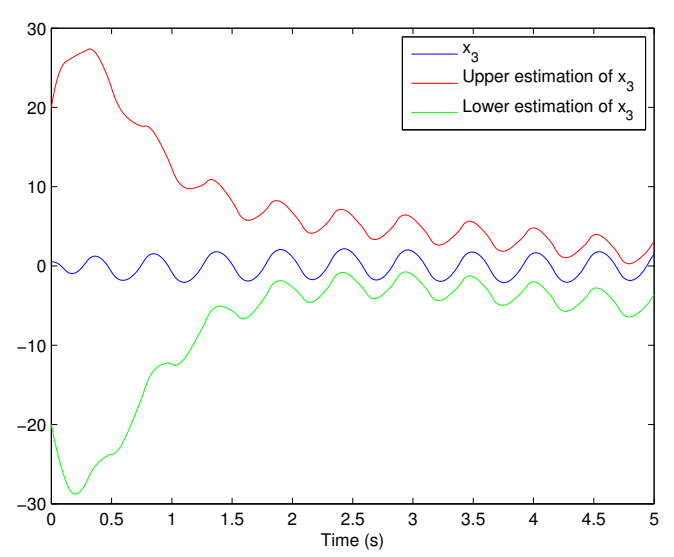

Figure 3: The interval estimation for $x_{3}$.

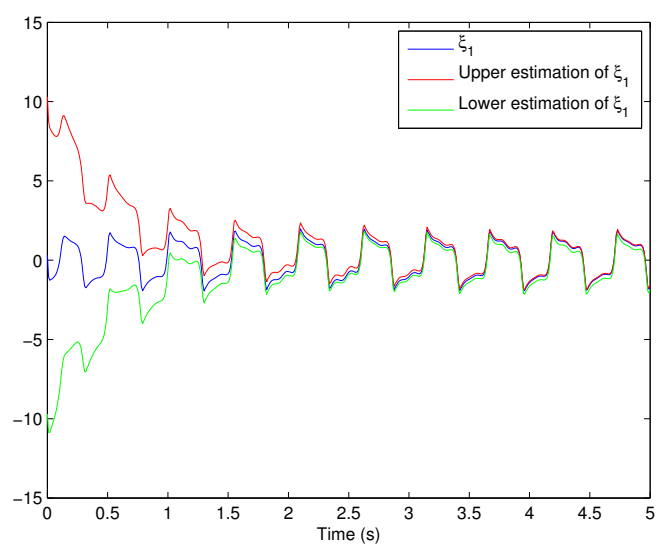

Figure 4: The interval estimation for $\xi_{1}$.

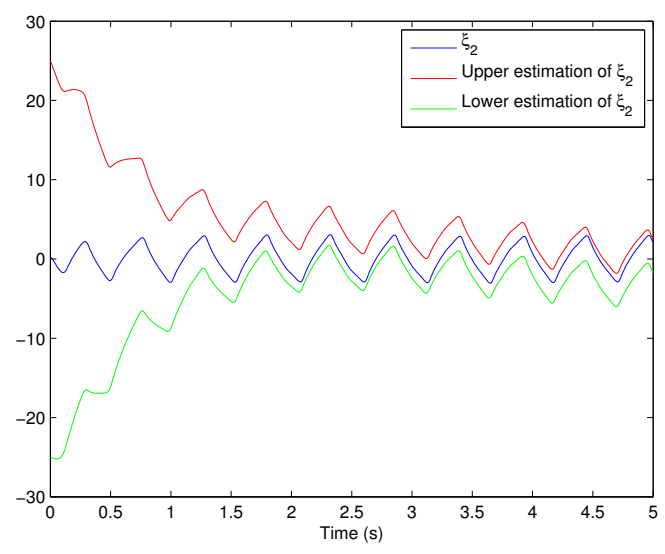

Figure 5: The interval estimation for $\xi_{2}$.

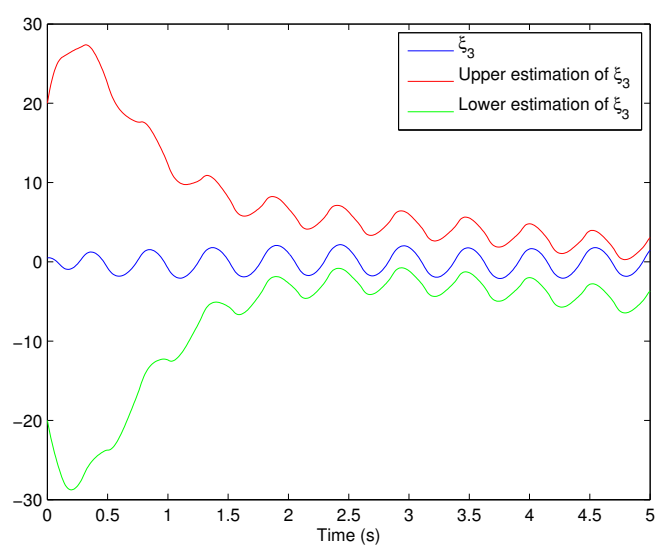

Figure 6: The interval estimation for $\xi_{3}$. 


\subsection{Physical example}

Consider the following nonlinear RLC circuit in Fig. 7, modified from Yang et al. (2009), where the capacitor is not linear,

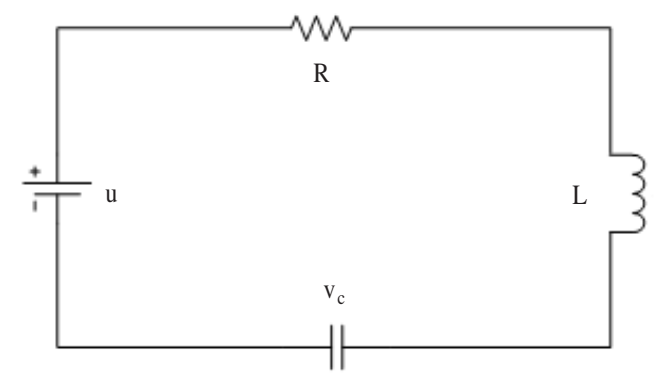

Figure 7: Nonlinear RLC ciruit.

but satisfying nonlinear $q-v_{c}$ characteristic $v_{c}=q+0.5 \sin q^{2}$. According to the analysis in Yang et al. (2009), we have

$$
\begin{aligned}
& \dot{q}=\phi / L \\
& \dot{\phi}=-\phi R / L-v_{c}+u \\
& 0=v_{c}-q-0.5 \sin q^{2} \\
& y=v_{c}
\end{aligned}
$$

where $u$ is source voltage, $q$ and $v_{c}$ represent respectively the charge and the voltage of the capacitor, and $\phi$ is the flux through the inductor. Denote $\xi=\left(q, \phi, v_{c}\right)^{T}$, and taking into account the uncertainties, the above dynamics can be written into the form (1) with

$$
\begin{aligned}
& \bar{E}=\left[\begin{array}{lll}
1 & 0 & 0 \\
0 & 1 & 0 \\
0 & 0 & 0
\end{array}\right], \bar{A}=\left[\begin{array}{ccc}
0 & 1 / L & 0 \\
0 & -R / L & -1 \\
-1 & 0 & 1
\end{array}\right] \text { and } \bar{C}=\left[\begin{array}{l}
0 \\
0 \\
1
\end{array}\right]^{T} \\
& \bar{f}(\xi, u)=\left[\begin{array}{c}
0 \\
0 \\
-0.5 \sin \left(\xi_{1}^{2}\right)
\end{array}\right] \text { and } v(t)=\left[\begin{array}{c}
0.2 \sin 2 t \\
0.2 \sin 4 t \\
0.1 \cos 3 t
\end{array}\right], w(t)=
\end{aligned}
$$

$0.1 \sin (t)$, where $v(t)$ and $w(t)$ are the assumed disturbances.

In the simulation setting, we choose $L=5, R=10$ and $u=1.5+30 \sin 2.8 t$. For such a simple nonlinear singular system, we can easily follow the proposed procedure to design an interval observer, thus the intermediate steps are omitted. The simulation results are depicted in Fig. 8-10.

\section{Conclusion}

When treating the uncertain nonlinear singular system while the asymptotic estimation is not possible, this paper is devoted to designing an interval observer for the studied uncertain system. By imposing the rank conditions and assuming the boundedness of the uncertainties (and the derivative of the uncertainty) in the state and in the output, we proved that an interval observer can always be synthesized to provide the upper and the lower estimations of the real state. The efficiency of the proposed method is highlighted by two examples.

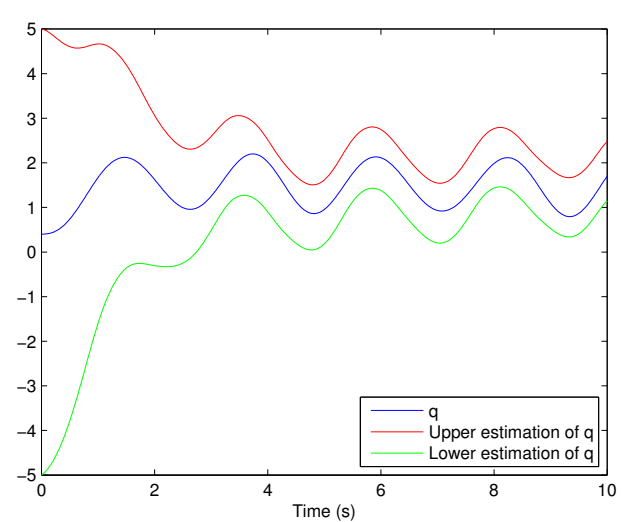

Figure 8: The interval estimation for $q$.

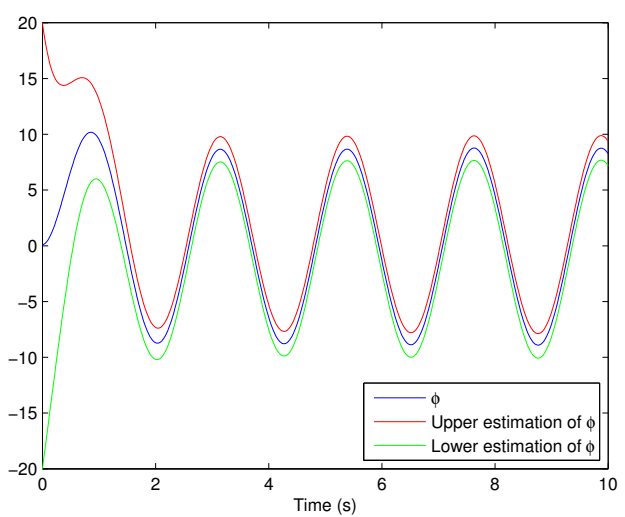

Figure 9: The interval estimation for $\phi$.

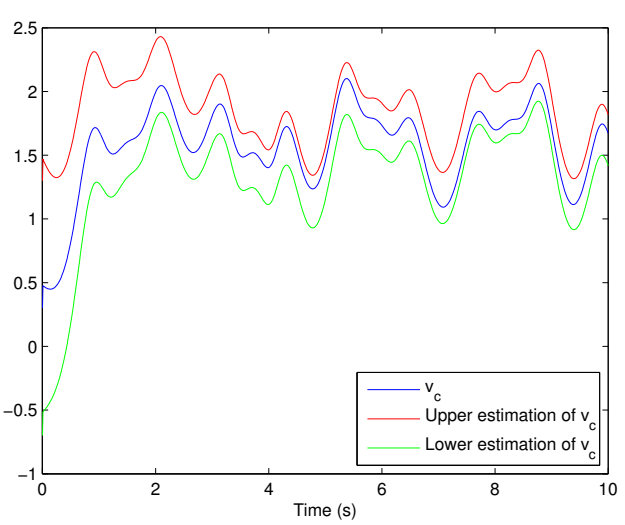

Figure 10: The interval estimation for $v_{c}$.

\section{References}


Bejarano, F., Floquet, T., Perruquetti, W., Zheng, G., 2011. Observability and detectability analysis of singular linear systems with unknown inputs. In: Decision and Control and European Control Conference (CDC-ECC), 2011 50th IEEE Conference on. pp. 4005-4010.

Bejarano, F., Perruquetti, W., Floquet, T., Zheng, G., 2012. State reconstruction of nonlinear differential-algebraic systems with unknown inputs. In: Decision and Control (CDC), 2012 IEEE 51st Annual Conference on. pp. 5882-5887.

Bejarano, F. J., Floquet, T., Perruquetti, W., Zheng, G., 2013. Observability and detectability of singular linear systems with unknown inputs. Automatica 49(3), 793-800.

Bejarano, F. J., Floquet, T., Perruquetti, W., Zheng, G., 2015. Observation of nonlinear differential-algebraic systems with unknown inputs. IEEE Transactions on Automatic Control 60(7), 1957-1962.

Bitsoris, G., 1978. Principle de comparaison et stabilité des systèmes complexes. Ph.D Thesis, Paul Sabatier University of Toulouse.

Borne, P., Dambrine, M., Perruquetti, W., Richard, J., 2003. Vector lyapunov functions: nonlinear, time-varying, ordinary and functional differential equations. Advances in Stability Theory at the End of the 20th Century. edited by AA Martynyuk, Taylor \& Francis.

Boutat, D., Zheng, G., Boutat-Baddas, L., Darouach, M., 2012. Observers design for a class of nonlinear singular systems. In: in Proc. IEEE Conf. Decision Control.

Boutayeb, M., Darouach, M., 1995. Observers design for non linear descriptor systems. In: in Proc. IEEE Conf. Decision Control.

Campbell, S., 1980. Singular systems of differential equations I. Pitman.

Campbell, S., 1982. Singular systems of differential equations II. Pitman.

Chebotarev, S., Efimov, D., Rassi, T., Zolghadri, A., 2015. Interval observers for continuous-time $\{\mathrm{LPV}\}$ systems with / performance. Automatica 58, 82 -89 .

Cobb, D., 1984. Controllability, observability, and duality in singular systems. IEEE Transactions on Automatic Control AC-29 (12), 1076-1082.

Dai, L., 1989. Singular Control Systems. Vol. 118. Lecture Notes in Control and Information Sciences, Springer-Verlag, New York.

Dambrine, M., Goubet, A., Richard, J. P., 1995. New results on constrained stabilizing control of time-delay systems. In: Proc. 34st IEEE Conference on Decision and Control.

Darouach, M., Boutat-Baddas, L., 2008a. Observer for a class of nonlinear singular systems. IEEE Trans.Autom.Contr 35, 2627-2633.

Darouach, M., Boutat-Baddas, L., 2008b. Observers for a class of nonlinear singular systems. IEEE Transactions on Automatic Control 53 (11), 2627 2633

Darouach, M., Boutayeb, M., 1995. Design of observers for descriptor systems. IEEE Trans. Automatic Control 40(7), 1323-1327.

Darouach, M., Zasadzinski, M., Hayar, M., 1996. Reduced-order observer design for descriptor systems with unknown inputs. IEEE Transactions on Automatic Control 41 (7), 1068-1072.

Efimov, D., Fridman, L., Raïssi, T., Zolghadri, A., Seydou, R., 2012. Interval estimation for lpv systems applying high order sliding mode techniques. Automatica 48, 2365-2371.

Efimov, D., Polyakov, A., Richard, J.-P., 2014. Interval estimation for systems with time delays and algebraic constraints. In: in Proc. of ECC.

Gouzé, J.-L., Rapaport, A., Hadj-Sadok, Z., 2000. Interval observers for uncertain biological systems. Ecological Model 133, 45-56.

Grujic̀, L. T., Martynyuk, A., Ribbens-Pavella, M., 1987. Large Scale Systems Stability under Structural Perturbations. Lecture Notes in Control and Information Sciences, Springer Verlag, New York

Hou, M., Muller, P., 1999a. Casual observability of descriptor systems. IEEE Transactions on Automatic Control 44 (1), 158-163.

Hou, M., Muller, P., 1999b. Observer design for descriptor systems. IEEE Transactions on Automatic Control 44 (1), 164-169.

Kaczorek, T., 2007. Polynomial and Rational Matrices Applications in Dynamical Systems Theory. Communications and control engineering. Springer.

Kamke, E., 1932. Zur theorieder systemegewohnlicher dierentialgliechungen ii. Acta Math 58, 57-85.

Kaprielian, S., Turi, J., 1992. An observer for a nonlinear descriptor system. in Proc. IEEE Conf. Decision Control.

Koenig, D., 2005. Unknown input proportional multiple-integral oberver design for linear descriptor systems: application to state and fault estimation. IEEE Transactions on Automatic Control 50 (2), 212-217.

Koenig, D., 2006. Observers design for unknown input nonlinear descriptor systems via convex optimization. IEEE Trans. Automatic Control 51(6), 1047-1052.

Koenig, D., Mammar, S., 2002. Design of proportional-integral observer for unknown input descriptor systems. IEEE Transactions on Automatic Control 47 (12), 2057-2062.

Lu, G., Ho, D., 2006. Full-order and reduced-order observer for lipschitz descriptor systems: The unified lmi approach. IEEE.Trans on Circuits and systems-II: Express Briefs 53, 563-567.

Mazenc, F., Bernard, O., 2010. Asymptotically stable interval observers for planar systems with complex poles. IEEE Tran 55(2), 523-527.

Müller, M., 1926. über das fundamental theorem in der theorie der gewöhnlichen differentialgleichungen. Math. Z, 619-645.

Paraskevopoulos, P., Koumboulis, F., August 1992. Observers for singular systems. IEEE Transactions on Automatic Control 37 (8), 1211 - 1215.

Perruquetti, W., Richard, J., 1996. Connecting wasewskis conditions with mmatrices: Application to constrained stabilization. Dynam. Syst. Appl 5, 81-96.

Perruquetti, W., Richard, J., Borne, P., 1995a. Vector lyapunov functions: Recent developments for stability, robustness, practical stability, and constrained control. Nonlinear Times and Digest 2, 227-258.

Perruquetti, W., Richard, J., Grujić, L. T., Borne, P., 1995b. On practical stability with the settling time via vector norms. International Journal of Control 62 (1), 173-189.

Raïssi, T., Efimov, D., Zolghadri, A., 2012. Interval state estimation for a class of nonlinear systems. IEEE Trans. Automatic Control 57, 260-265.

Smith, H., 1995. Monotone dynamical systems: An introduction to the theory of competitive and cooperative systems. Surveys and Monographs, AMS, Providence 41

Wazewski, T., 1950. Systèmes des équations et des inégalités différentielles ordinaires aux seconds membres monotones et leurs applications. Ann. Soc. Polon. Math 23, 112-166.

Yang, C., Zhang, Q., Chai, T., 2009. Observer design for a class of nonlinear descriptor systems. In: in Proc. IEEE Conf. Decision Control.

Yip, E., Sincovec, R., 1981. Solvability, controllability, and observability of continuous descriptor systems. IEEE Transactions on Automatic Control AC-26 (3), 702-707.

Zheng, G., Efimov, D., Perruquetti, W., 2013. Interval state estimation for uncertain nonlinear systems. In: IFAC, NOLCOS

Zheng, G., Efimov, D., Perruquetti, W., 2016. Design of interval observer for a class of uncertain unobservable nonlinear systems. Automatica 63, $167-$ 174 